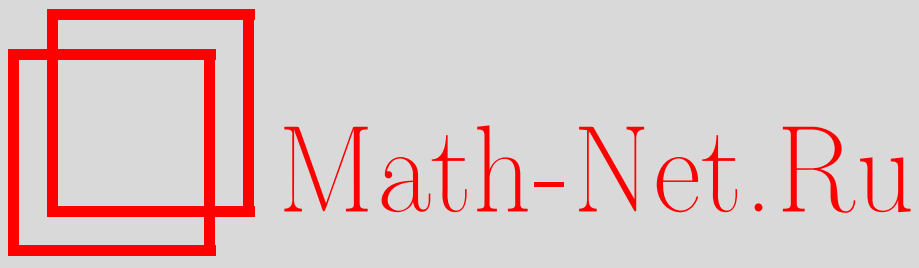

С. А. Никищенков, Диагностическая модель реконфигурируемых систем на основе информационно-логических схем процессов, Вестн. Сам. гос. техн. ун-та. Сер. Физ.-мат. науки, 2005, выпуск 34, 119-127

DOI: https://doi.org/10.14498/vsgtu345

Использование Общероссийского математического портала Math-Net.Ru подразумевает, что вы прочитали и согласны с пользовательским соглашением http: //www.mathnet.ru/rus/agreement

Параметры загрузки:

IP : 54.198 .187 .58

26 апреля 2023 г., 15:20:45

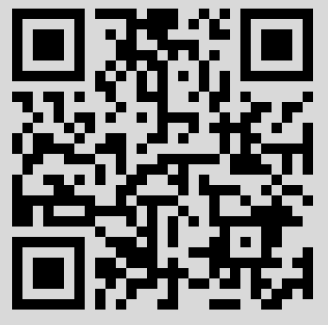




\section{ДИАГНОСТИЧЕСКАЯ МОДЕЛЬ РЕКОНФИГУРИРУЕМЫХ СИСТЕМ НА ОСНОВЕ ИНФОРМАЦИОННО-ЛОГИЧЕСКИХ СХЕМ ПРОЦЕССОВ}

Рассмотрены информационно-логические схемы процессов в качестве модели для функционального диагностирования систем с переменной конфигурацией. Проведен анализ свойств информационнологических схем в обнаружении дефектов в параллельном потоке операций по сравнению с графсхемами алгоритмов. Исследованы теоретические и прикладные аспекты применения модели $в$ задачах диагностики технологических систем.

Постановка задачи. Под конфигурацией системы будем понимать распределение дискретных операций процесса обработки исходных информационно-материальных носителей в результат согласно принятой технологии, в структуре с параллельно работающими взаимодействующими взаимозаменяемыми функциональными блоками и во времени $[1,2]$. Реконфигурация - это факт перехода системы от одной конфигурации к другой, т.е. структурновременное перераспределение операций в их параллельном потоке, вызванное изменением порядка следования операций в блоках, переходом от последовательных алгоритмов управления к параллельным и от статических методов управления к динамическим, применением новых тактик использования ресурсов и стратегий жизнедеятельности. К причинам реконфигурации относятся: асинхронность и конвейеризация выполнения операций; распараллеливание технологии (и обратный процесс - уменьшение степени параллелизма); деградация системы вследствие отказов части блоков; новая диспетчеризация (регламент, сценарий) или самоорганизация системы; динамическое распределение ресурсов и операций; изменения стратегий по производительности и живучести и т.д. [3-7]. Для класса производственно-технологических реконфигурируемых систем (РС) задача функционального диагностирования (ФД), т.е. контроля правильности работы при выполнении предписанных функций, приобретает особую значимость, так как система существенно усложняется, растет поток дефектов (по их типу и интенсивности), последствия которых приводят к значительным информационно-материальным потерям, и оказываются неэффективными известные методы ФД. При этом первоочередной становится задача выбора или разработки диагностической модели (ДМ), учитывающей особенности объекта ФД и обладающей преимуществами перед известными моделями.

Сравнительный анализ и выбор диагностической модели. Обобщенные условия правильного функционирования (УПФ) РС могут быть представлены путем интерпретации фундаментальных свойств алгоритмических систем из [8] в виде требований к процессам обработки (см. табл.1). Соответственно невыполнение этих условий (т.е. исключение из правил) задает достаточно полный список признаков дефектов (ПД) [5,9].

Т а б л и ц а 1

Условия правильного функционирования и признаки дефектов на системном уровне

\begin{tabular}{|c|l|l|}
\hline № & \multicolumn{1}{|c|}{ Условия правильного функционирования } & \multicolumn{1}{|c|}{ Признаки дефектов } \\
\hline 1 & $\begin{array}{l}\text { Процесс избирателен: определен на конечном множестве } \\
\text { операций и предикатов }\end{array}$ & $\begin{array}{l}\text { Выполняется операция не из } \\
\text { заданного допустимого множества }\end{array}$ \\
\hline 2 & $\begin{array}{l}\text { Процесс упорядочен: задан технологией, алгоритмом, его } \\
\text { распараллеленной формой или иным методом }\end{array}$ & $\begin{array}{l}\text { Нарушен порядок выполнения } \\
\text { операций }\end{array}$ \\
\hline 3 & $\begin{array}{l}\text { Процесс результативен: приводит к требуемому } \\
\text { преобразованию исходных материалов в конечную } \\
\text { продукцию }\end{array}$ & Операции не приводят к результату \\
\hline 4 & $\begin{array}{l}\text { Процесс своевременен: имеет начало и предельные } \\
\text { времена выполнения операций, этапов и всей технологии } \\
\text { Времени не выполняется операция, } \\
\text { этап или технология }\end{array}$ \\
\hline
\end{tabular}

Цель моделирования с точки зрения диагноста - это представление поведения РС в условиях возникновения и проявления сбоев и отказов оборудования, неверных действий персонала, ошибок проектирования, непредусмотренных условий эксплуатации и других 
факторов. Традиционно под ДМ объекта ФД понимается совокупность его формальной функциональной модели, УПФ и ПД [9-11]. ДМ предназначена для адекватного и достаточного описания и анализа объекта ФД и разработки способов, алгоритмов и средств ФД. Это осуществляется путем преобразования УПФ и ПД в выражения, пригодные для проверки средствами ФД в процессе работы. Переход от ДМ к УПФ и ПД характеризует способ ФД, а алгоритмы обнаружения дефектов и средства ФД разрабатываются на основе принятого способа и требований к системе ФД. Данная методология широко применяется, например, для ФД вычислительных систем на разных уровнях - от диагностирования цифровых схем до оперативного контроля взаимодействующих процессов.

Методы ФД технических систем основаны на введении избыточности в объект диагностирования и классифицируются по следующим признакам: ориентация на проверку операции или блока; тип диагностической модели и класс обнаруживаемых дефектов; состав и форма контролирующих соотношений; способ сжатия информации об объекте; реализация в виде глобальных или локальных средств; степень перенастраиваемости средств ФД; необходимость и способ преобразования объекта к контролепригодному виду. Под эффективностью понимается мера обнаруживающей способности средств ФД по отношению к заданным дефектам при ограничениях на аппаратную, временную и информационную избыточность. При сравнительном анализе ДМ следует учитывать как теоретические аспекты (универсальность, полнота, разрешимость и проработанность основных формальных задач анализа, синтеза и преобразования, преемственность), так и инженерные задачи моделирования (интерпретируемость, наглядность, операбельность, эффективность базовых процедур, унифицируемость). Положительно оцениваются простота процедур интерпретации объекта, развитость методик синтеза и анализа ДМ, приемлемая размерность описания, реализуемость процедур по сжатию модели для обеспечения эффективности диагностирования, наглядность форм представления. Это означает целесообразность использования известных алгоритмических и графовых форм представления.

По сравнению со структурно-ориентированными методами и средствами, методы ФД по функциональным моделям обладают преимуществами: возможностью ФД на макроуровне, что соответствует интересам собственника; меньшей избыточностью для достижении заданной эффективности; низкой зависимостью от вариантов технической реализации.

Метод ФД по схемам алгоритмов предложен в $[10,12]$ и заключается в следующем:

диагностируемый объект признается бездефектным, если правильно выполняется предписанный прикладной алгоритм обработки, заданный в виде граф-схемы алгоритма (ГСА), например на рис. $1, a$;

исходя из ГСА общего вида, список дефектов содержательно включает: пропадание оператора, замена оператора на другой, запуск более одного оператора, неправильный переход по значению логического условия (ЛУ);

дефекты возникают в произвольный момент времени и на любом шаге алгоритма;

контролирующие соотношения, соответствующие ГСА выполняемого алгоритма и списку дефектов, оперативно проверяются средствами ФД по потоку диагностических признаков, поступающих с объекта ФД;

исходный алгоритм подвергается преобразованию, сохраняющему функциональную эквивалентность, с целью приведения к контролепригодному виду и повышения эффективности ФД.

Основное применение ФД по ГСА нашло для специализированных вычислительных и автоматизированных систем с последовательной архитектурой.

Для ФД параллельных автоматизированных систем со статическим распараллеливанием алгоритмов метод нашел развитие в [13-15], где в качестве ДМ выступают параллельные ГСА (ПГСА), имеющие специальные операторы распараллеливания, объединения и ожидания fork, join и wait, с достаточно полным списком дефектов: в ветвях ПГСА - аналогично дефектам на ГСА; запуск операторов не во всех ветвях после оператора fork; преждевременный запуск операторов после join или wait. Пример ПГСА приведен на рис.1, 6 .

Процесс реализации ПГСА с теоретико-множественной точки зрения есть прохождение по ПГСА как ориентированному графу и запуск подмножества операторов на основании известного подмножества выполненных в предыдущих тактах операторов и текущих значений ЛУ. Дефект «неправильный переход по значению ЛУ» представляется как «ложный запуск оператора», а дефекты на ПГСА, характерные операторам fork, join и wait, сводятся к нему же или к пропаданию оператора. Таким образом, справедлива возможность сведения списка 120 
дефектов на ПГСА к следующему: пропадание оператора, замена оператора на другой и ложный запуск оператора.
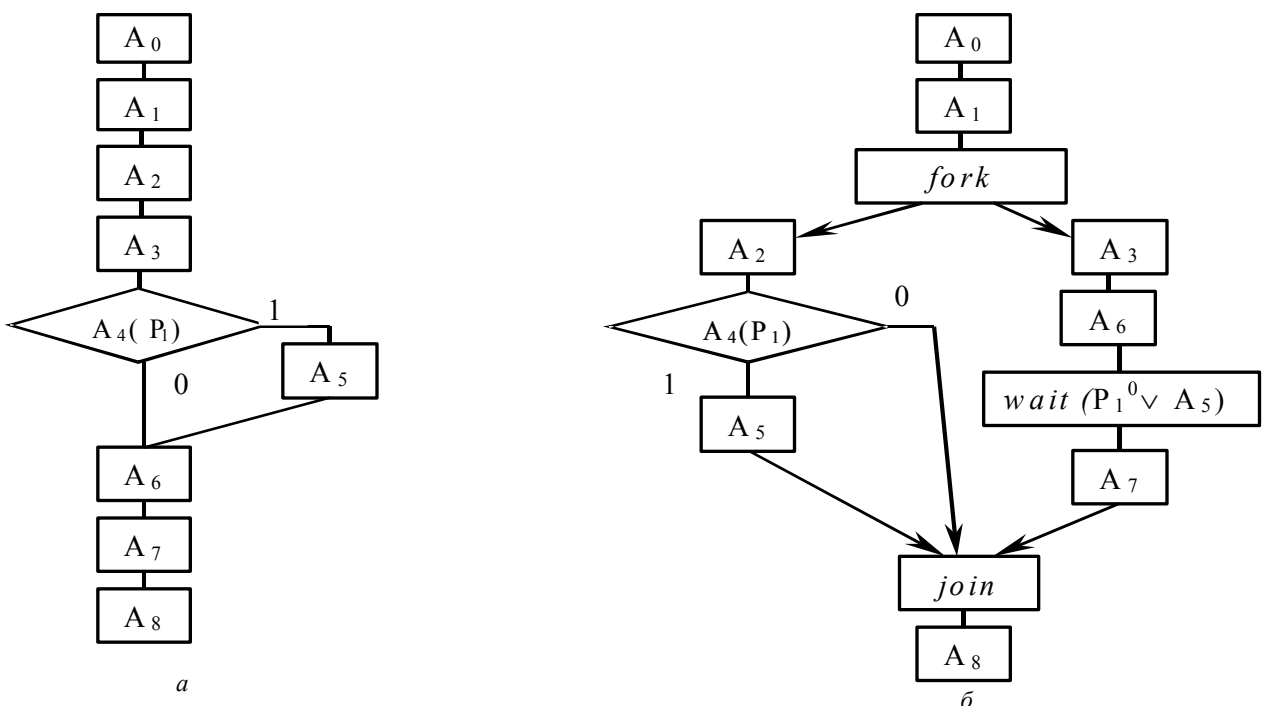

Р и с. 1. Эквивалентные ГСА:

$a$ - последовательная, $\sigma$ - параллельная со специальными операторами

Указанные положения определяют свойства, преимущества и недостатки систем ФД по схемам алгоритмов. Метод носит фундаментальный характер, поскольку базируется на категории и свойствах алгоритмических систем $[8,16]$, и универсален по отношению к классу объектов, функционирование которых описывается классическими схемами алгоритмов. Для ГСА разрешимы все теоретические проблемы, а также задачи интерпретации. Использование схем алгоритмов рационально для описания УПФ и ПД на уровне операций технологических систем, где в полной степени проявляются практически все виды дефектов.

К недостаткам схемно-алгоритмического подхода (относительно поставленной задаче ФД РС) относятся большая размерность ДМ при описании фиксированного множества конфигураций РС; неприменимость для РС с динамическим распараллеливанием; отсутствие описания информационной составляющей процесса обработки в ДМ; ориентация на обнаружение дефектов в управляющей части объекта ФД.

К числу моделей параллельных вычислений, описывающих на операторном уровне выполнение вычисления с произвольным распараллеливанием, относятся А-схемы Котова Нариньяни [17-20]. Спусковые функции представляют собой конечный предикат над множеством операторов и ЛУ в схеме программы (включая в общем случае дополнительные, учитывающие историю процесса) и задают максимально параллельный асинхронный процесс выполнения (для класса так называемых «свободных» схем), обеспечивая запуск операторов по мере готовности их входных аргументов. Управление по спусковым функциям предполагает наличие соответствующей архитектуры и механизмов управления в системе [18]. Метод спусковых функций, реализующих проверку возможности запуска операторов на множестве допустимых параллельных процессов, представляется идеальным механизмом контроля правильности, однако рассматривался в качестве ДМ для систем со статическим распараллеливанием $[14,15]$.

Среди моделей теоретического программирования, описывающих информационную составляющую вычислительного процесса, следует отметить информационный граф, информационно-логическую графовую историю, схемы информационных связей и т.д. $[2,21,22]$. Они отличаются описанием внутреннего параллелизма вычисления и множества реализаций алгоритма с различной степенью распараллеливания. Интерпретация операторов в них совпадает с интерпретацией операторов в соответствующих ПГСА, при этом известны формализованные процедуры синтеза, анализа, графовой декомпозиции и преобразования. Диагностические модели, учитывающие информационную составляющую процесса, ориентированы на обнаружение искажений при выполнении последовательных программ и тестирование параллельных программ. Перспективная модель потоков данных [18] обладает 
существенной сложностью и большим допустимым списком дефектов при ее использовании в качестве ДМ.

Для ДМ широкого назначения возможно применение сетей Петри [21,22]. Свойства данной модели в смысле охвата объектов различного вида (алгоритмы и программы, аппаратура, протоколы и т.д.) вытекают из допустимого произвола в интерпретации ее элементов. Однако практическое использование сетей Петри затруднено из-за неоднозначности и большой размерности описаний реальных объектов и сложности синтеза и анализа.

К числу графовых моделей, наглядно описывающих варианты параллельных процессов, относятся билогические графы (БГ) [23-25], которые использовались для расчета характеристик и планирования работы мультипроцессорных вычислительных систем. Как показано в [15], при их применении в качестве ДМ РС удовлетворяется большинство требований, перечисленных выше. К числу вопросов, требующих специального рассмотрения при разработке ДМ на основе БГ, относятся: определение отношений между схемами алгоритмов и программ и БГ (вопросы интерпретации); свойства БГ, общие и отличные по сравнению с моделями параллельных вычислений; соотношения между дефектами в РС и дефектами, задаваемыми на данной ДМ (обнаруживающие или диагностические свойства модели).

Определение, содержание, формы представления и общие свойства ИЛСП. Пусть задана технология (алгоритм) преобразования информационно-материального базиса в виде упорядоченной совокупности операторов (преобразователей и распознавателей) с указанием кортежей их входных и выходных переменных, т.е. операторная схема программы (рис. 2,a). Тогда информационно-логической схемой процесса (ИЛСП) назовем тройку $\mathbf{S}=(\mathbf{A}, \mathbf{L}, \mathbf{F}), \mathbf{B}$ которой:

множество $\mathbf{A}=\left\{A_{i}\right\}, \quad i=\{0,1, \ldots, k\}$ состоит из операторов алгоритма (преобразователей и распознавателей), причем для распознавателей в скобках указывается проверяемое условие из множества проверяемых ЛУ алгоритма $\mathbf{P}=\left\{P_{j}\right\}, j=\{1,2, \ldots, l\}$, например $A_{i}\left(P_{j}\right)$;

множество предикатов $\mathbf{L}=\mathbf{P} \cup \mathbf{Q}$ включает множество ЛУ алгоритма $\mathbf{P}$ и множество дополнительных предикатов $\mathbf{Q}$, характеризующих историю процесса выполнения алгоритма;

множество $\mathbf{F}=\left\{F_{i}(\mathbf{A}, \mathbf{L})\right\}$ представляет совокупность логических функций в базисе алгебры Жегалкина (И, Исключающее ИЛИ, 1), означающих событие выполнения операторовпредшественников из А для оператора $A_{i}$ при заданных значениях предикатов $\mathbf{L}$.

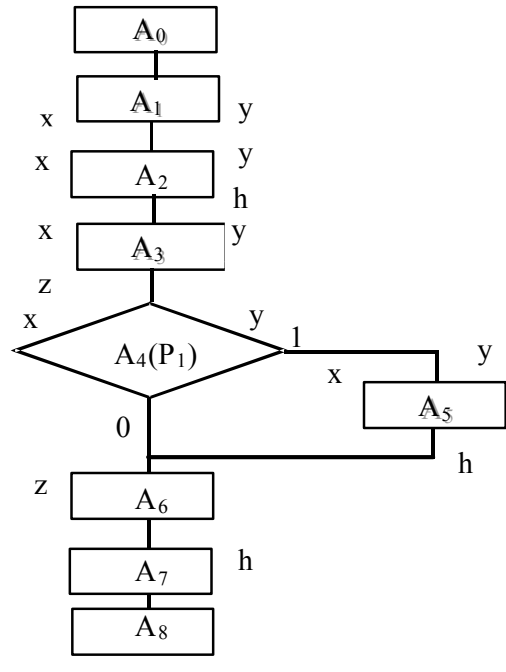

$a$

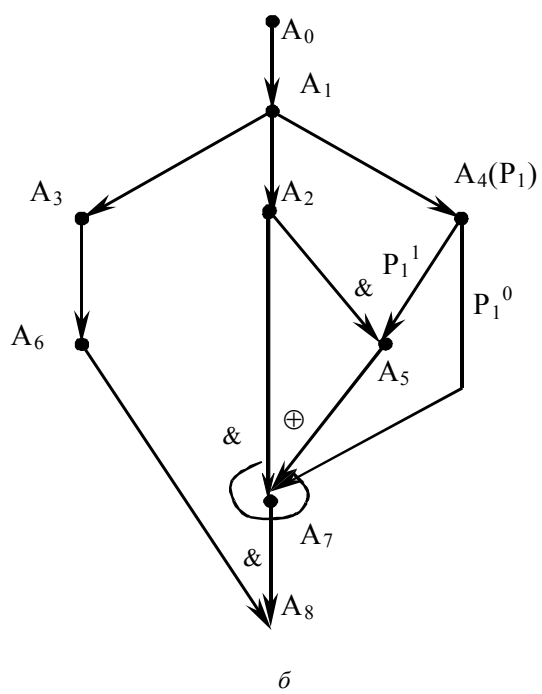

Р и с. 2. К определению ИЛСП:

$a$ - схема программы, $\sigma$ - ИЛСП в виде билогического графа

Строковая форма записи ИЛСП есть список условий правильного запуска операторов (операторных УПФ) вида $A_{i} \leftarrow F_{i}(\mathbf{A}, \mathbf{L})$, где функции И и Исключающее ИЛИ обозначаются символами \& и $\oplus$.

Для схемы на рис.2,a, она имеет следующий вид:

$A_{1} \leftarrow A_{0} ; A_{2} \leftarrow A_{1} ; A_{3} \leftarrow A_{1} ; A_{4} \leftarrow A_{1} ; A_{5} \leftarrow A_{4}\left(P_{1}{ }^{l}\right) \& A_{2} ; A_{6} \leftarrow A_{3} ; A_{7} \leftarrow\left(A_{2} \& A_{4}\left(P_{1}{ }^{0}\right)\right) \oplus A_{5} ; A_{8} \leftarrow A_{6} \& A_{7}$ 
Например, для. $A_{7}$ запуск считается верным (оператор может выполниться), если выполнились $A_{2}$ и $A_{4}\left(\right.$ со значением $\left.P_{1}=0\right)$, или выполнились $A_{5}$ и $A_{4}\left(\right.$ со значением $\left.P_{1}=1\right)$.

Таким образом, ИЛСП описывает совокупность операторов-предшественников в информационном и логическом смысле для каждого из операторов процесса и содержит единственное и конечное правильное условие его запуска в зависимости от хода процесса при произвольном (асинхронном параллельном) порядке запуска операторов. В этом отношении принципиальным отличием ИЛСП от схем управления (при любом методе управления - от алгоритмического до максимально параллельного) является описание допустимого, а не необходимого порядка запуска операторов, т.е. отсутствие детерминизма.

Графически ИЛСП представляется билогическим орграфом $\mathrm{G}=(\mathbf{A}, \mathbf{B}, \mathbf{L}, \&, \oplus)$, достаточным для полного описания строковой формы, где A - множество вершин; $\mathbf{B}$ - множество дут, интерпретирующих отношения логического и информационного предшествования между операторами, такое, что $B_{i, k}=1$ для вершин $A_{i}$ и $A_{k}$, если $A_{i}$ входит аргументом в $F_{k}$; каждая вершина $A_{i}$ размечена входной логикой $F_{i}$ с помощью связок $\&, \oplus$ между входящими дугами; каждая вершина, соответствующая оператору-распознавателю $A_{i}\left(P_{j}\right)$, размечена выходной логикой с помощью связки $\oplus$ между альтернативными выходящими дугами, нагруженными различными значениям $P_{j}\left(P_{j}^{l}\right.$ или $\left.P_{j}^{0}\right)$, и связкой $\&$ между дугами, нагруженными одинаковыми значениям $P_{j}$.

Примеры ИЛСП даны на рис. 2,б и рис. 3, где приняты допущения: по умолчанию действует логика И, символ которой при разметке может опускаться; связка по логическим функциям между дугами может уточняться вспомогательной соединительной линией.

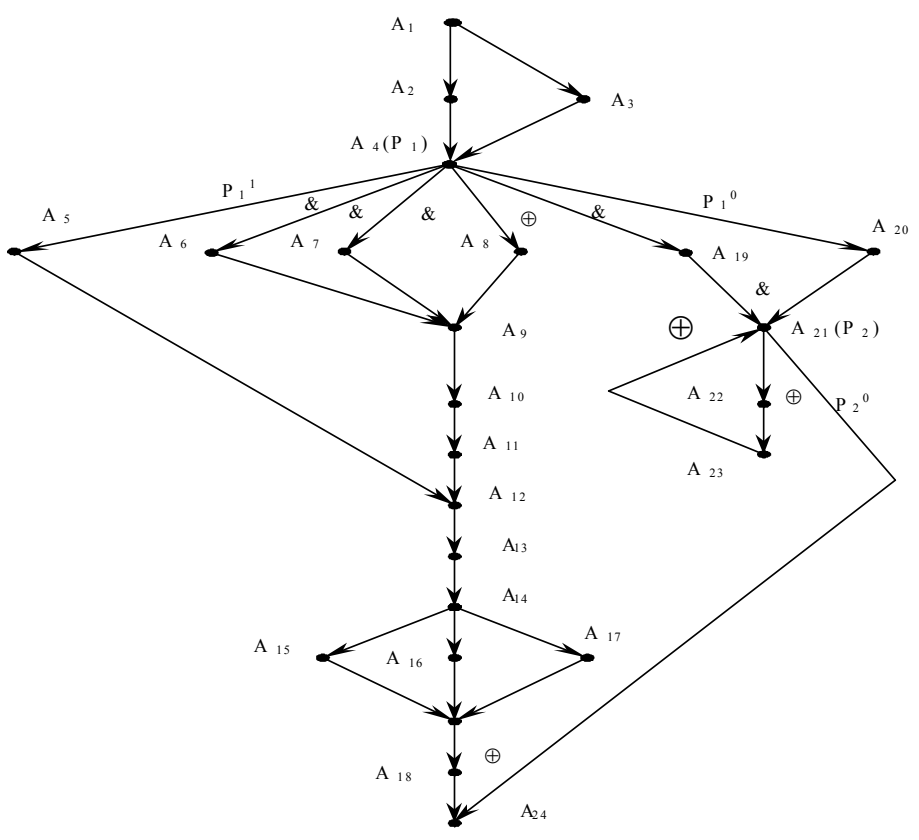

Р и с. 3. ИЛСП диагностики и ремонта подсистемы подачи топлива в дизеле

Согласно [22] ИЛСП в общем случае является простым непланарным слабосвязанным правильным управляющим графом со специальной разметкой вершин и дуг, семантика которого служит целям потокового анализа систем.

Динамика ИЛСП как контролирующей сети может быть описана как процесс, состоящий из таких событий, как «включение» вершин и «зажигание» дуг [23-25]:

процесс начинается включением начальной вершины и заканчивается выключением конечной;

включение вершин означает готовность к выполнению соответствующего оператора;

выполнение операторов задается внешним по отношению к ИЛСП потоком управления (в том числе с дефектами); 
после выполнения оператора, соответствующего включенной вершине, зажигаются ее выходные дуги с истинными значениями предикатов, а вершина выключается;

вершина включается, если входные дуги зажжены соответственно входной логике, причем после ее включения они гасятся;

в процессе выполнения до момента выключения конечной вершины множество включенных вершин и зажженных дут не пусто.

Как показано в [14], важнейшим свойством ИЛСП является естественная возможность моделирования конвейерных процессов, для чего достаточно разрешить включение вершин и выполнение операторов вновь, не дожидаясь включения конечной вершины.

Для заданных значений ЛУ выполнение ИЛСП моделируется конъюнктивным графом (с входной и выходной И-логикой для всех вершин), аналогичным по содержанию последовательности операторов при выполнении граф-схемы алгоритмов [16,24]. Конъюнктивный граф получается путем удаления дуг, предикат на которых имеет альтернативное значение, и вершин, для которых удалены все входные дуги. Ациклической ИЛСП соответствует конечный набор конъюнктивных графов, для циклических БГ возможно построение конъюнктивных графов развертыванием циклов и заданием кратности их повторения. Важным свойством конъюнктивных графов является возможность формальной проверки корректности БГ по критериям принадлежности любой вершины хотя бы одному конъюнктивному графу [16,24].

Свойства диагностической модели на основе ИЛСП. Правильное выполнение ИЛСП характеризуется тем, что множество запускаемых операторов $\mathbf{A}_{t}^{3}$ есть непустое подмножество «готовых» операторов $\mathbf{A}_{t}^{\mathbf{r}}$, у которых выполнены условия запуска:

$$
\left(\forall t \exists \mathbf{A}_{t}^{3} \subset \mathbf{A}_{t}^{\mathbf{\Gamma}}\right) \wedge\left(\left|\mathbf{A}_{t}^{3}\right|>=1\right)=1 .
$$

Нарушение данного УПФ представляется следующими ПД на ИЛСП:

$$
\left(\exists t \exists A_{i, t} \not \subset \mathbf{A}_{t}^{\mathbf{r}}\right) \vee\left(\left|\mathbf{A}_{t}^{3}\right|=0\right)=1,
$$

т.е. на ИЛСП заданы 2 типа дефектов - «ложный запуск» и «отсутствие запуска».

Совокупность конкретной ИЛСП и соотношений (1) и (2) назовем ДМ на основе ИЛСП. В [15] доказана эффективность применения ДМ на основе ИЛСП для ФД параллельных деградирующих систем, реализующих множество ПГСА с различной степенью распараллеливания, при этом вначале определялись отношения вычисляемости между множеством ПГСА с произвольной степенью распараллеливания и соответствующей ИЛСП, а затем доказывалась покрываемость дефектов на ПГСА дефектами на ИЛСП. ПГСА есть упорядочение над множеством операторов в ходе реализации схемы, под которой понимается процесс формирования потока операторов схемы для заданных значений ЛУ. Аналогичное справедливо для ИЛСП, с той разницей, что в ИЛСП нет директивного указания на запуск операторов. При определении отношений между ИЛСП и множеством ПГСА, различающихся степенью распараллеливания, примем, что процессу запуска операторов ПГСА однозначно соответствует запуск операторов-преобразователей и распознавателей в ИСА. Для определения диагностических свойств формировался следующий ряд утверждений.

1. Перестановки операторов схемы программы, независимых по условию Бернштейна Рассела - Нариньяни, сохраняют эквивалентность по информационному графу и функциональную эквивалентность.

2. Статическое распараллеливание схем программ не изменяет отношения информационного и логического предшествования между операторами при произвольных реализациях.

3. Переход от А-схемы к ее производной есть дополнительное упорядочение условий запуска операторов и сохраняет функциональную эквивалентность при произвольных реализациях схем.

4. При правильном выполнении ПГСА соблюдаются условия запуска операторов ИЛСП,

5. Для множества эквивалентных ПГСА, определенных на совпадающих множествах операторов-преобразователей и распознавателей и различающихся степенью распараллеливания, ИСА является инвариантом при произвольной правильной реализации любой ПГСА;

6. Дефекты «замена» и «ложный запуск» на ПГСА необходимо проявляются как дефект «ложный запуск» на ИЛСП, причем это происходит в том же такте при условии непринадлежности заменяемого или ложно запускаемого оператора множеству «готовых» операторов, либо в следующих тактах при условии принадлежности данному множеству. 
7. Дефект «пропадание» оператора на ПГСА необходимо проявляется в текущем такте как дефект «отсутствие запуска» на ИЛСП, если больше не запускается ни один оператор, либо как дефект «ложный запуск» на ИЛСП в следующих тактах в остальных случаях.

По отношению к другим (нестатическим) методам управления диагностические свойства ИЛСП могли быть установлены с помощью понятия инварианта, под которым в теории схем программ и теории параллельного программирования понимается объект, относительно которого рассматриваемые схемы или их реализации эквивалентны по результату или истории процесса [17,20-22]. В работах [19,20] показано, что инвариантом может быть информационный граф, относительно которого распознается эквивалентность цепочек операторов в реализации схемы и тем самым эквивалентность самих схем программ. Вместе с тем, для возможных диаграмм процесса запуска операторов производной А-схемы КотоваНариньяни эквивалентность по информационному графу не соблюдается [18]. Сказанное ставит задачу определения отношений эквивалентности на множестве реконфигурируемых потоков операторов, различающихся методом и степенью распараллеливания, и установления возможности использования ИЛСП в качестве их инварианта, однако она является алгоритмически неразрешимой [22]. Приведем ряд утверждений о правильных процессах, реконфигурации, ИЛСП и обнаружении дефектов для ограниченного класса РС, в котором не применяется принцип «расклейки» операторов и функция упреждения [18].

1. Для технологии, имеющей внутренний параллелизм, существует множество эквивалентных потоков операций, различающихся конфигурацией, под которой понимается структурно-временное распределение операций согласно применяемого метода управления (программирования, диспетчеризации) и стратегии жизнедеятельности технологической системы.

2. Любая конфигурация потока операций (в том числе классическая алгоритмизация и статическое распараллеливание) есть дополнительное структурно-временное упорядочение максимально параллельного потока операций.

3. Перестановка независимых операций, переход от одной степени распараллеливания потока к другой или динамическое распараллеливание операций приводит к его реконфигурации, т.е. к структурно-временному перераспределению операций в системе.

4. Для каждой РC заданного класса существует максимально параллельный (асинхронный) поток операций, соответствующий управлению по спусковым функциям, т.е. совпадающий с ИЛСП:

$$
\forall t \forall A_{i, t} \subset \mathrm{A}_{t}^{3}=\mathrm{A}_{t}^{\mathrm{r}},
$$

являющийся инвариантом к множеству (пространству) конфигураций РС.

5. Обнаружение дефектов по ИЛСП применимо для любой конфигурации потока операций и, следовательно, в условиях его реконфигурации, т.е. ИЛСП является диагностическим инвариантом РС.

6. При использовании ФД РС по ИЛСП обладает решается задача оперативного обнаружения дефектов в потоке операций, существенных по отношению к искажению информационно-материального базиса.

7. Оперативность обнаружения дефектов, понимаемая как задержка от момента появления дефекта до его проявления на ИЛСП, тем выше, чем меньше разность $\left|\mathbf{A}_{t}^{\mathbf{r}}\right|-\left|\mathbf{A}_{t}^{3}\right|$, т.е. чем больше степень распараллеливания обработки.

Интерпретации, синтез, анализ и классификация ИЛСП. В зависимости от представления алгоритма, технологии или процесса работы РС на неформальном или формальном языках, задача построения ИЛСП сводится к формализации или к трансляции (интерпретации), аналогично задаче построения операторных схем алгоритмов [10,16]. Рассмотрение вопросов синтеза и интерпретации ИЛСП позволяет сделать важный вывод о том, что построение ИЛСП базируется на схемной интерпретации дискретных процессов и является составной частью задачи распараллеливания, поэтому для этого могут быть использованы известные приемы и средства, в частности, автоматизированные средства распараллеливания алгоритмов и программ [26,27].

Построение ациклических ИЛСП по схеме программы осуществляется согласно методике статического распараллеливания программ, где ИЛСП является промежуточным результатом распараллеливания $[18,19,26]$. Для схем программ процедура построения ИЛСП заключается в следующем. На первом этапе строится граф зависимостей между операторами алгоритма на основании перебора возможных выполнении схемы и анализа информационных и логических 
связей между операторами. На втором осуществляется нагружение дуг графа значениями ЛУ, формирование условий запуска операторов (строчной формы ИЛСП) и их минимизация (путем обнаружения и исключения транзитивных зависимостей). На заключительном этапе по строчной форме ИЛСП строится БГ с проверкой на корректность с использованием конъюнктивных графов.

Для циклических схем программ построение ИЛСП обладает особенностями формирования условий запуска операторов, исходя из условий статического или динамического распараллеливания циклов [18,26] .

Оценка и классификация ИЛСП целесообразна на основе методов анализа схем программ и орграфов [17,18] с учетом специфики использования ИЛСП как ДМ РС. Количественные параметры ИЛСП по размерности, логической обусловленности и связности позволяют сравнивать ИЛСП с точки зрения приспособленности для реализации того или другого способа обнаружения дефектов при ФД РС [28].

Классификация ИЛСП по типам информационно-логических структур базируется на работах [2-4,23]. Для процессов вычислительного характера анализ внутреннего параллелизма задач показал, что матричным вычислениям соответствуют ИЛСП с только конъюнктивной логикой, а к ациклическим ИЛСП с произвольной логикой относятся практически все, получаемые при параллельной реализации различных способов внутренней сортировки, фильтрации и т.п. В производственных РС ИЛСП имеют прозвольный характер, определенный технологией обработки и ее информационно-логической структурой. Анализ РС в сфере железнодорожного транспорта позволяет определить такие типичные примеры ИЛСП, как Идеревья (сборка-разборка агрегатов, аналитические задачи), чередующиеся узлы с конъюнктивной и дизъюнктивной логикой (диагностика и ремонт подвижного состава), регулярные слабосвязанные маршруты (оперативное управление перевозками) и т.д.

Применение ДМ на основе ИЛСП. Теоретические ограничения на применение метода ФД по ИЛСП связаны с одной стороны, с проблемой максимального параллелизма в процессах и допустимого пространства реконфигураций, а с другой - с возможностью статического описания РС в виде конечного набора ИЛСП. Как показано в [2,3,18,22] при максимальном распараллеливании условие запуска оператора схемы программы общего вида может иметь сколь угодно сложный вид. Это вытекает из-за вариаций информационных и логических предшественников при произвольном развитии процесса. Предложенные ИЛСП, описывающие допустимый порядок запуска операторов алгоритма, в случае применения механизмов распараллеливания, близких к максимальному, необходимо включают предикаты Q, представляющие соотношения между числами запусков операторов и характеризующие тем самым историю процесса. В этом случае можно говорить об интерпретации ИЛСП счетчиковыми схемами, для которых доказана возможность максимально параллельной реализации операторной схемы произвольного вида $[18,20]$. В работах $[19,20]$ показано, что для класса приводимых схем программ условие запуска оператора конечно, и может быть построена А-схема, максимально асинхронная для исходной схемы программы.

Эволюция методов параллельной компьютерной обработки данных [18,21] в направлении получения предельно быстрых вычислений привело к применению принципов «расклейки» операторов и тотального упреждения их выполнения, что является существенно избыточным по использованию ресурсов и нерационально для технологических РС. Тем самым применимость метода ФД по ИЛСП остается в силе для широкого класса РС производственнотехнологической сферы.

Практические ограничения накладываются степенью контролепригодности РС, характеризуемой в основном возможностью доступа к информации о составе и порядке операций в технологии и снятия диагностических признаков с блоков РС.

Применение метода ФД по ИЛСП целесообразно по следующим направлениям:

организация систем контроля и диагностики ответственных высокопроизводительных технологий с изменяющимися стратегиями [5];

изучение внутреннего параллелизма технологий и сбалансированности процессов в РС; [14].

разработка однородных диагностических процессоров, близких по функциям к нейросетям

Для обеспечения эффективности ФД над ИЛСП могут быть выполнены следующие процедуры:

вертикальная (траекторная ) и горизонтальная (ярусная) декомпозиции для разработки способов и алгоритмов обнаружения дефектов [15,28]; 
обогащение модели свертками или ассоциативными признаками функций и времен выполнения операторов для создания универсальной диагностической сетевой модели РС;

разбиения на части и изоморфные фрагменты, гомоморфное сжатие для повышения эффективности ФД [22].

\section{БИБЛИОГРАФИЧЕСКИЙ СПИСОК}

1. Хорошевский В.Г. Инженерный анализ функционирования вычислительных машин и систем. М.: Радио и связь, 1987. 256c.

2. Алгоритмы, математическое обеспечение и архитектура многопроцессорных вычислительных систем. Под ред. А.П.Ершова. М.: Наука, 1982, 336 с.

3. Параллельные вычислительные системы с общим управлением. И.В. Прангишвили и др. М.: Энергоатомиздат, 1983. $312 \mathrm{c}$.

4. Валях Е. Последовательно-параллельные вычисления. М.: Мир, 1985. 456 с.

5. Никищенков C.A. Проблемы функционального диагностирования реконфигурируемых систем управления // Исследования и разработки ресурсосберегающих технологий на железнодорожном транспорте: Межвуз. сб. науч. тр. Вып. 23. Самара: СамГАПС, 2002. С.93-96.

6. Харченко В.С. Модели и алгоритмы реконфигурации отказоустойчивых систем с адаптивной многоярусной мажоритарно-резервированной структурой // Автоматика и телемеханика. 2000. №12. С.162-175.

7. Никищенков С.A. Функциональное диагностирование реконфигурируемых информационно-управляющих систем // Информационные технологии на железнодорожном транспорте (Инфотранс-2002). 7-я междунар. науч.-практ.конф. СПб: ПГУПС, 2002. С. 172.

8. Кузнецов О.П., Адельсон-Вельский Г.М. Дискретная математика для инженера. М.: Энергоатомиздат, 1988.

9. Основы технической диагностики: Модели объектов, методы и алгоритмы диагноза / Под ред. П.П.Пархоменко. М.: Энергия, 1976. С. 272 с.

10. Смолов В.Б., Барашенков В.В., Балакин В.Н. Контролирующие и диагностические процедуры на схемах алгоритмов. // Тез. докл. V Всесоюз. совещ. по проблемам управления. Ч.3. М.: Наука, I971. С.95-96.

11. Щербаков Н.С., Подкопаев Б.П. Структурная теория контроля цифровых автоматов. М.: Машиностроение, I982. $191 \mathrm{c}$.

12. Усачев Ю.Е. Функциональное диагностирование управляющей части ЭВМ по граф-схемам алгоритмов: Дис. ... канд. техн. наук. Л.: ЛЭТИ, 1984, 152 с.

13. Балакин В.Н., Барашенков В.В., Казак А.Ф. и Никищенков С.А. Устройство для контроля параллельной вычислительной системы. А.с. № 1451697, 1989, бюл. №2.

14. Балакин В.Н., Барашенков В.В., Казак А.Ф. и Никищенков С.А. Устройство для контроля блоков управления. А.c. № 1365986 (СССР), 1988, бюл. №1.

15. Никищенков С.A. Функциональное диагностирование управляющей части реконфигурируемых многопроцессорных вычислительных систем по информационным схемам алгоритмов. Дис. ... канд. техн. наук. Л.: ЛЭТИ, 1988, 156 с.

16. Барашенков В.В. Анализ и преобразования операторных схем алгоритмов. Л.: ЛЭТИ, 1979. 103 с.

17. Котов В.E. Введение в теорию схем программ. Новосибирск: Наука, 1978. 268 с.

18. Элементы параллельного программирования / В.А.Вальковский, В.Е.Котов, А.Г.Марчук, Н.Н. Миренков // Под ред. В.Е.Котова. М.: Радио и связь, I983. 240 с.

19. Нариньяни А.С. Теория параллельного программирования: формальные модели // Кибернетика. I974. №3. C.1-15; №4. C.1-14..

20. Котов В.Е., Нариньяни А.С. Асинхронные вычислительные процессы над памятью // Кибернетика. I966. №3. C.64-71.

21. Бунич А.Л. и др. Параллельные вычисления и задачи управления (аналитический обзор) // Автоматика и телемаханика, 2002. №12. С. 3-23.

22. Касьянов В.Н., Евстигнеев В.А. Графы в программировании: обработка, визуализация и применение. СПб.: БХВ - Петербург, 2003. $1104 \mathrm{c}$

23. Головкин Б.А. Расчет характеристик и планирования параллельных вычислительных процессов. М: Радио и связь, 1983. $272 \mathrm{c.}$

24. Рыжкков А.П. Правильная билогическая граф-модель параллельного вычислительного процесса и его свойства // Изв. АН СССР. Техническая кибернетика, 1976, №2. С.96-I04.

25. Baer J.L., Bovet D.P., Estrin G. Legality and other properties of graph models of computations // J. ACM, 1987. V. 17. N3. P.543-554.

26. Вальковский В.А., Котов В.Е. Автоматическое построение параллельных программ. Новосибирск: 1975.95 с.

27. Барбан А.П., Игнатущенко В.В. Распараллеливание структурированных программ // Электронное моделирование. 1982. №2. С.28-35.

28. Никищенков С.А. Способы функционального диагностирования управляющих систем по информационнологическим схемам процессов. М.: ВНТИЦ, 2001. №73200100203.

Поступила 15.12.2003 2. 